\title{
Image-Based Device Tracking for the Co-registration of Angiography and Intravascular Ultrasound Images
}

\author{
Peng Wang ${ }^{1}$, Terrence Chen ${ }^{1}$, Olivier Ecabert ${ }^{2}$, Simone Prummer ${ }^{2}$, \\ Martin Ostermeier ${ }^{2}$, and Dorin Comaniciu ${ }^{1}$ \\ 1 Siemens Corporate Research, Siemens Corporation, Princeton NJ, U.S.A \\ 2 Siemens AG, Healthcare Sector, Siemensstr. 1, Forchheim, Germany
}

\begin{abstract}
The accurate and robust tracking of catheters and transducers employed during image-guided coronary intervention is critical to improve the clinical workflow and procedure outcome. Image-based device detection and tracking methods are preferred due to the straightforward integration into existing medical equipments. In this paper, we present a novel computational framework for image-based device detection and tracking applied to the co-registration of angiography and intravascular ultrasound (IVUS), two modalities commonly used in interventional cardiology. The proposed system includes learning-based detections, modelbased tracking, and registration using the geodesic distance. The system receives as input the selection of the coronary branch under investigation in a reference angiography image. During the subsequent pullback of the IVUS transducers, the system automatically tracks the position of the medical devices, including the IVUS transducers and guiding catheter tips, under fluoroscopy imaging. The localization of IVUS transducers and guiding catheter tips is used to continuously associate an IVUS imaging plane to the vessel branch under investigation. We validated the system on a set of 65 clinical cases, with high accuracy (mean errors less than $1.5 \mathrm{~mm}$ ) and robustness (98.46\% success rate). To our knowledge, this is the first reported system able to automatically establish a robust correspondence between the angiography and IVUS images, thus providing clinicians with a comprehensive view of the coronaries.
\end{abstract}

\section{Introduction}

In image guided interventions, accurate and robust localization of medical devices can provide valuable information to improve clinical workflow and to facilitate the operations in interventions. In this paper, we present a computational framework for medical device detection and tracking in X-ray images, and demonstrate it with a novel application for the co-registration of $2 \mathrm{D}$ angiography and intra-vascular ultrasound (IVUS) images. The framework is briefly illustrated in Fig. 1. The X-ray angiography is the primary modality that guides percutaneous interventions (PCI), providing the spatial structure information of coronaries. The IVUS images can provide rich information on the vessel wall 


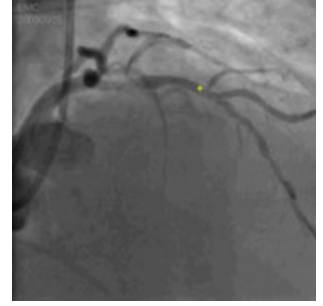

(a)

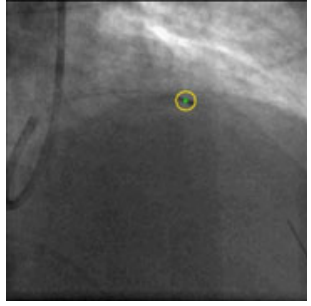

(b)

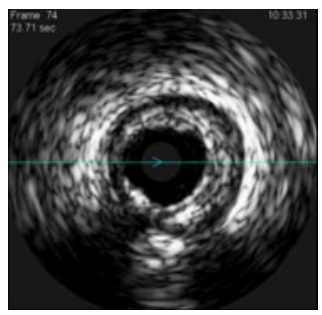

(c)

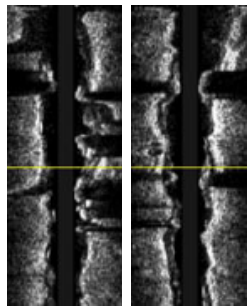

(d)

Fig. 1. Device tracking for the co-registration of IVUS and angiography images. (a): a angiogram frame. The yellow cross indicate a registered IVUS imaging plane; (b): a fluoroscopic frame. The yellow circle represents a tracked IVUS transducer; $(c)(d)$ : cross-sectional and axial view of IVUS images. The yellow lines in the axial views indicate the registered IVUS image.

composition and extent of plaques, being used for tissue characterization, the analysis of lesion, and the estimation of stent expansion. However, the lack of the vessel orientation information makes it difficult to fully understand the spatial structure of vessels. The co-registration of the two image modalities will combine the high spatial resolution from the IVUS data with the good overview and $2 \mathrm{D} / 3 \mathrm{D}$ orientation obtained from angiography, facilitating the usage of IVUS in cathlab and improving clinical outcome of cardiac interventions [1,2].

To register the two image modalities, we need to find the position of IVUS image plane along a vessel branch during the IVUS pullback. Manual labeling, as adapted in few preliminary systems [13], is straightforward. But manually finding the correspondences between IVUS and angiography data needs expertise and can be time-consuming. Furthermore, without continuous tracking, previous work inevitably assumes a constant IVUS pullback speed to estimate IVUS imaging planes from an interpolation 3.5. This paper presents a computational framework for automated image-based IVUS transducer detection and tracking in X-ray images to continuously identify the location of IVUS image plane, and to achieve an unconstrained co-registration of angiography and IVUS images.

Accurate and robust tracking in X-ray is challenging. The devices undergo cardiac motion and breathing motion during interventions. Even with ECG triggering, the breathing motion could still be large. Considering the low signal-tonoise ratio of fluoroscopy images and the presence of other surgical devices in the images, conventional tracking methods based on intensity appearance or edgebased energy would encounter difficulties. We present a framework that employs learning-based detections and model-based probabilistic tracking to address the aforementioned challenges. The learning-based detectors detect various devices present in this application, i.e., IVUS transducers, the body and tips of a guiding catheter in which the IVUS transducer is inserted, and the wire that is used to pull the IVUS transducers. A probabilistic model-based tracking framework is introduced to combine detections of different devices in a Bayesian inference framework, to achieve robust and accurate tracking. The device tracking results 
are then used for a geodesic distance based registration to associate an IVUS imaging plane at each time stamp during pullback with a point on the coronary branch under investigation. The detection and tracking is automated, only requiring the selection of a coronary branch in angiography as an input. It does not assume a constant IVUS pullback speed, and can work under different projection angles. We validate the framework on a set of 65 clinical cases acquired in hospitals. Quantitative evaluation results show that the framework has achieved high tracking robustness and accuracy for the co-registration of IVUS and angiography images.

\section{Workflow}

As illustrated in Fig. 2, the system workflow includes two stages: the "angiogram" stage and the "pullback" stage. In the angiogram stage, contrast agents are injected to visualize vessel branches. A frame at the end-diastole (ED) phase is used to select the vessel branch under investigation where the IVUS imaging will take place, as the input to subsequent tracking and registration. A vessel detection method that requires minimal user interactions [7] is applied to segment the center line of a vessel branch and to extend the centerline toward the guiding catheter. In the pullback stage, an IVUS transducer is pulled back from the distal end of the target vessel branch toward the proximal end. During the pullback, both the fluoroscopic images, which are trigged by ECG at the ED cardiac phase, and IVUS images are acquired with time stamps. Due to the existence of continuous breathing motion, an additional point other than the IVUS transducer is needed as the reference point to compensate the breathing motion. The tip of a guiding catheter, through which the IVUS transducer is inserted and pull, is selected as the reference point, as it is the most stable and distinguishable point that we can find in the fluoroscopy. In the pullback stage, our method automatically detects and tracks the movements of IVUS transducers and guiding catheter tips in fluoroscopy, and maps the tracked IVUS transducers back to the selected vessel branch. Simultaneously the IVUS images are registered to the vessel, by using the time synchronization between two image modalities.

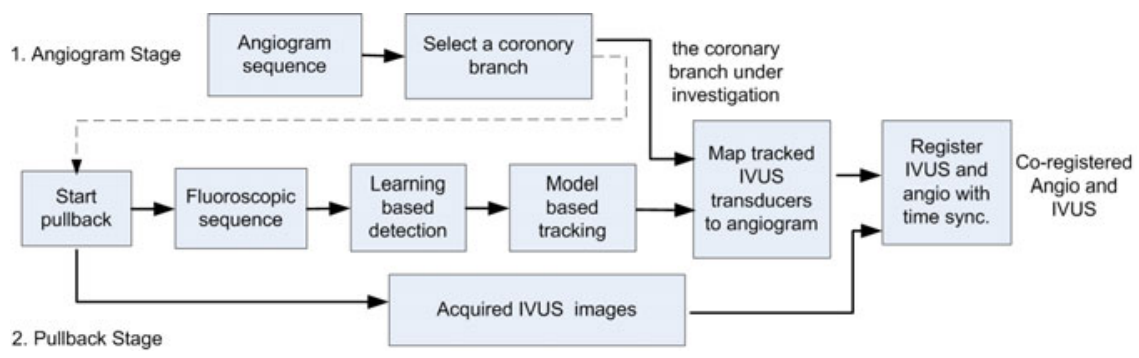

Fig. 2. The co-registration system workflow 


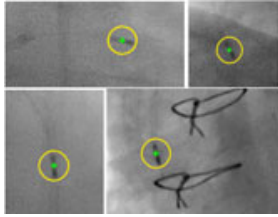

(a)

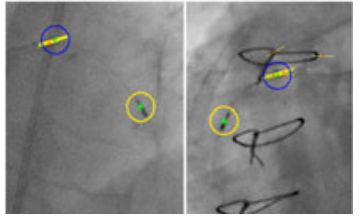

(b)

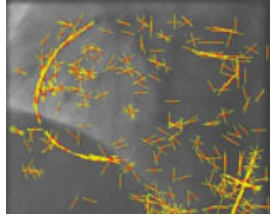

(c)

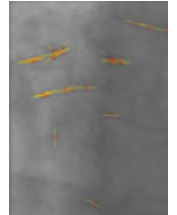

(d)

Fig. 3. Learning-based detection examples. (a): yellow circles represent detected IVUS transducers; (b): blue circles represent detected guiding catheter tips. Here multiple detection results close to each other are clustered into a single detection result; (c): detected segments of wire; (d) detected segments of guiding catheter body.

\section{Learning-Based Device Detection}

Learning-based detectors are trained from a set of off-line collected data, including both object samples (positive) and non-object samples (negative), to learn the decision boundary that separates different classes. In this work, the probabilistic boosting tree (PBT) and Haar features [4 are used to train the learning-based detectors. Here, we denote the probabilistic outputs from PBT as $P(\mathbf{Z} \mid \mathbf{x})$, where $\mathbf{Z}$ is the observed image, and $\mathbf{x}$ is the object state (i.e., if an image patch belongs to the positive or negative class.) Four types of detectors are trained. They are denoted as $P_{\text {IVUS_trans }}, P_{\text {cath_body }}, P_{\text {cath_tip }}$ and $P_{\text {wire }}$, for the IVUS transducer, the guiding catheter body, the guiding catheter tip, and the wire body respectively. Note that the guiding catheter body and wire detectors aim at the detection of segments, not the whole structure. Some detection results are shown in Fig. 3, Due to image artifacts and low visibility of devices, there exist false detections. To make the tracking robust to detection errors, a tracking framework that integrates multiple detections is introduced in the Section 4

\section{Model-Based Device Tracking and Registration}

\subsection{A Bayesian Tracking Framework}

Since the fluoroscopic images are acquired by ECG triggering, the device mainly undergoes breathing motion, which can be approximated by affine motion. The breathing motion at the $t$-th frame is denoted as $M_{t}=\left(m_{t}^{x}, m_{t}^{y}, m_{t}^{r}\right)$, where $m_{t}^{x}$, $m_{t}^{y}$, and $m_{t}^{r}$ are the $2 \mathrm{D}$ translation and rotation parameters. The motion tracking is expressed as the inference of the motion parameters from the fluoroscopic sequences acquired at the pullback stage. We formalize the parameter inference in a sequential Bayesian inference framework. Assuming a commonly used Markov property for tracking, the posterior probability $P\left(\mathbf{M}_{t}=\left(m_{t}^{x}, m_{t}^{y}, m_{t}^{r}\right) \mid \mathbf{Z}_{t}\right)$ is given in Eqn. (11):

$$
P\left(\mathbf{M}_{t} \mid \mathbf{Z}_{t}\right) \propto P\left(\mathbf{M}_{t}\right) P\left(\mathbf{Z}_{t} \mid \mathbf{M}_{t}\right)
$$




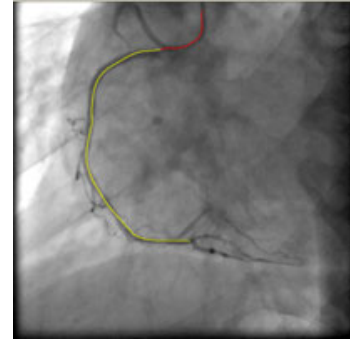

(a)

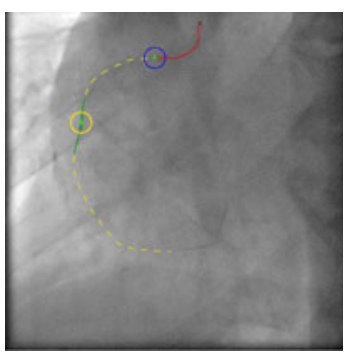

(b)

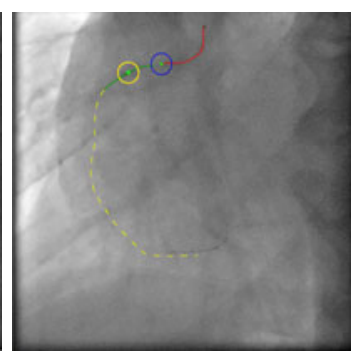

(c)

Fig. 4. Model-based tracking. (a): a vessel detected in the angiogram phase; (b)(c): an integrated model of point and curve elements is automatically initialized by the input from the angiogram stage and continuously tracked in the pullback stage.

where $\mathbf{Z}_{t}$ is an observed fluoroscopic sequence. The tracking result is the motion parameter corresponding to the maximal posterior probability, i.e. $\hat{\mathbf{M}}_{t}=$ $\arg \max P\left(\mathbf{M}_{t} \mid \mathbf{Z}_{t}\right)$. In Eqn. (1), $P\left(\mathbf{M}_{t}\right)$ is the prior probability. We model the $\mathbf{M}_{t}$

prior probability as $P\left(\mathbf{M}_{t}\right)=G\left(\mathbf{M}_{t} ; \Sigma_{\mathbf{M}}\right)$ where $G\left(\mathbf{M}_{t} ; \Sigma_{\mathbf{M}}\right)$ is a Gaussian model with a zero mean and the covariance matrix $\Sigma_{\mathbf{M}}$. The Gaussian prior model is used because of its simplicity and effectiveness of imposing smoothness constraints for 2D motions. Another component in Eqn. (1), the likelihood model $P\left(\mathbf{Z}_{t} \mid \mathbf{M}_{t}\right)$ measures the likelihood of motion parameters. In our method, the measurement of the model is a fusion of measurements of multiple devices, with more details provided in Section 4.2 .

\subsection{Integrated Model and Fusion of Individual Device Measurements}

Due to the low image quality of fluoroscopy and cluttered backgrounds, independently tracking each device is prone to detection errors. To improve the accuracy and robustness of tracking, we combine all the devices into an "integrated IVUS model". We denote such a model as $\Gamma_{t}$, in which each component is represented by a curve (e.g., a segment of guiding catheter body and a wire) or a point (e.g., guiding catheter tip and IVUS transducer), as shown in Fig 4, By defining the integrated model, we can re-write the likelihood in the form of curve representations, as $P\left(\mathbf{Z}_{t} \mid \mathbf{M}_{t}\right)=P\left(\mathbf{Z}_{t} \mid \Gamma_{t}\right)$. Based on the integrated IVUS model, the measurement model is a combination of measurements of individual components. For simplicity, we assume that the measurement of individual component is independent of each other given the curve, i.e., $P\left(\mathbf{Z}_{t} \mid \Gamma_{t}^{k}, \Gamma_{t}\right)=P\left(\mathbf{Z}_{t} \mid \Gamma_{t}^{k}\right)$ in which $\Gamma_{t}^{k}$ denotes a component in the integrated model. Therefore, we can further decompose the measurement model $P\left(\mathbf{Z}_{t} \mid \Gamma_{t}^{k}\right)$ as Eqn. (2):

$$
P\left(\mathbf{Z}_{t} \mid \mathbf{M}_{t}\right)=P\left(\mathbf{Z}_{t} \mid \Gamma_{t}\right)=\sum_{k} P\left(\mathbf{Z}_{t} \mid \Gamma_{t}^{k}\right) P\left(\Gamma_{t}^{k} \mid \Gamma_{t}\right) .
$$


The component measurements $P\left(\mathbf{Z}_{t} \mid \Gamma_{t}^{k}\right)$ are from the four detectors, i.e. $P_{\text {IVus_trans }}, P_{\text {cath_body }}, P_{\text {cath_tip }}$ and $P_{\text {wire }} \cdot P\left(\Gamma_{t}^{k} \mid \Gamma_{t}\right)$ defines the weight of individual component in the model, denoting the confidence of individual measurements. Such weights are set empirically in our method.

The integrated model to be tracked is initialized from the vessel detection at the angiogram phase. As shown in Fig. 4, the vessel detection identifies the vessel centerline, which is used to approximate the shape of wire. The guiding catheter body and tip is also initialized from the vessel detection. The IVUS transducer is initialized from the detection at the first frame in fluoroscopy. During tracking, the model is propagated from a previous frame, and updated from the motion parameter estimation. For computational efficiency, we apply a kernel-based multi-resolution method in the tracking 6 to achieve a speed of 1.0 frame per second in a computer with a Core 2 Duo 2.0GHz CPU.

\subsection{Registration}

The registration steps assume first the mapping of all tracked IVUS transducers to their corresponding positions along a vessel branch in angiography, and then the association of each IVUS image to the corresponding position in angiography. The first mapping between tracked IVUS transducers and selected vessel branch is based on the geodesic distance computed from the device tracking. The Euclidean distance between an IVUS transducer and a guiding catheter tip is directly computed from the tracking results, and then the geodesic distances can be inferred from the Euclidean distances based on the vessel shape. A constraint that the geodesic distance should change smoothly between successive frames is imposed to resolve potential ambiguity. Based on geodesic distances, each tracked IVUS transducer is mapped to a point on the vessel branch. After that, the association of IVUS images and angiography image is straightforward, with the use of the synchronized acquisition time of fluoroscopic and IVUS images.

\section{Experiments}

The method and system are evaluated on a set of clinical cases acquired from three different European hospitals. The acquisition follows the workflow described in Section 2. The evaluation set includes 65 cases. Each acquired case includes an angiogram sequence and a fluoroscopic pullback sequence (Artis Zee, Siemens AG, Forchheim, Germany), and corresponding IVUS images (Volcano Corp., San Diego, CA, U.S.A). The X-ray image size is 512 by 512 , and the physical size of each pixel is between $0.2 \mathrm{~mm}$ and $0.3 \mathrm{~mm}$. Each pullback takes a few minutes, usually containing 100 to 250 fluoroscopy frames. The data set includes a variety of clinical scenes, including low signal-to-noise ratio, different vessel branches and projection angles, and the existence of other surgical devices. Some examples are shown in Fig. 5 .

To establish ground truth for quantitative evaluation, we manually annotate the positions of IVUS transducers and guiding catheter tips in the set as the 

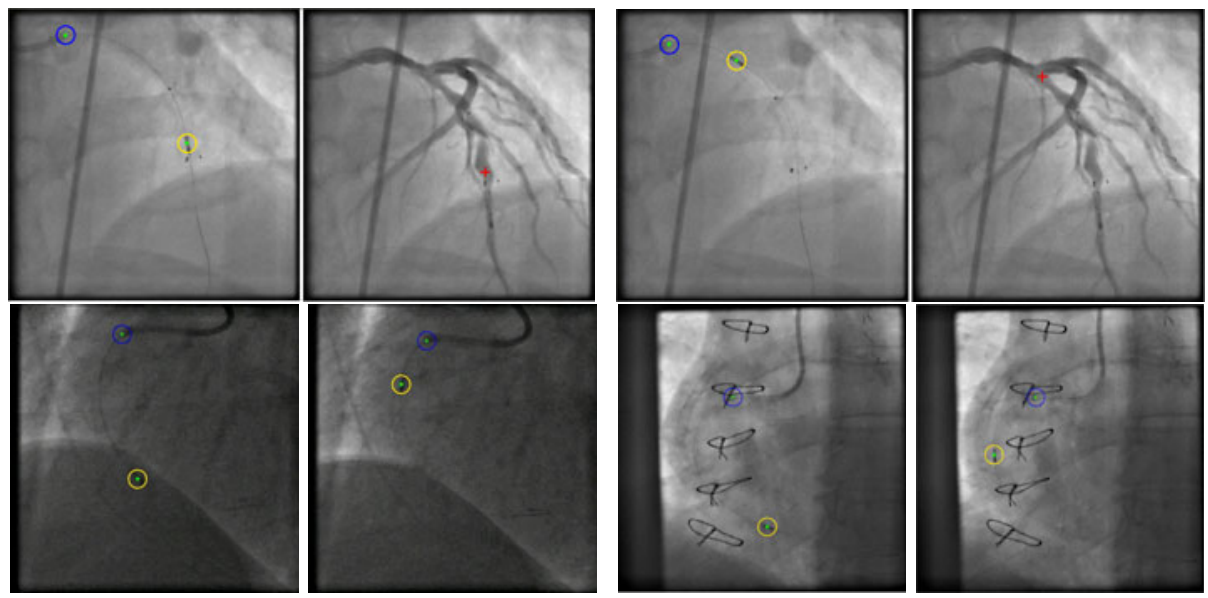

Fig. 5. Exemplar results: in each image of the first row, tracking results in the fluoroscopy are shown at the left, and the corresponding registration results in the angiogram are shown at the right; the second row shows more tracking results in fluoroscopy. IVUS images are not shown due to space limit.

ground truth. The tracking accuracy in fluoroscopy are quantitatively measured by the Euclidean distances between automatic tracking results and corresponding manual annotations. We further compute the geodesic distance error as the estimation of the registration error, because the final registration is based on the computed geodesic distance. For this purpose, we compute the geodesic distance from the manual annotations as the ground truth, and then compare this ground truth with the geodesic distance derived from corresponding automated tracking.

The quantitative validation of the system is performed with two experiments, i.e., Experiment 1 and Experiment 2. In the Experiment 1, the detectors are trained with and applied to all the cases. In the Experiment 2, the training and testing sets are separated to test how well our method can be generalized to unseen data. We train the detectors in 32 randomly selected cases, and validate the framework in the remaining 32 cases that are excluded from training. Our method is successful in 64 out of 65 cases, except for one case where the IVUS transducer is occluded by spines most time, thus achieving a $98.46 \%$ success rate. Some exemplar tracking results are shown in Fig. 5, Table 1 summarizes the error statistics from the 64 cases. The mm errors are converted from pixel errors based on the physical resolution of imaging detector, therefore quantifying normalized errors in a 2D projection space. The tracking error of IVUS transducers is small, with mean errors only around $0.35 \mathrm{~mm}$ for both experiments. The error at the guiding catheter tips is slightly larger. The median error is $0.97 \mathrm{~mm}$ for the Experiment 1, and $1.17 \mathrm{~mm}$ for the Experiment 2. The overall small differences between the two experiments demonstrate the good generalization capability of our tracking method. The mean geodesic errors are below $1.20 \mathrm{~mm}$ and $1.50 \mathrm{~mm}$ 
Table 1. Quantitative evaluation of the co-registration system

\begin{tabular}{|l|c|c|c||c|c|c|}
\hline Validation & \multicolumn{3}{|c||}{ Experiment 1 } & \multicolumn{3}{c|}{ Experiment 2 } \\
\hline Error & median & mean & std & median & mean & std \\
\hline IVUS transducer (in pixels) & 0.90 & 1.18 & 1.89 & 0.94 & 1.22 & 1.17 \\
\hline IVUS transducer (in mm) & 0.26 & 0.34 & 0.56 & 0.28 & 0.35 & 0.32 \\
\hline Guiding catheter tip (in pixels) & 3.34 & 5.43 & 6.42 & 4.10 & 6.72 & 7.43 \\
\hline Guiding catheter tip (in mm) & 0.97 & 1.57 & 1.82 & 1.17 & 1.92 & 2.09 \\
\hline Geodesic distance (in pixels) & 2.53 & 4.16 & 7.67 & 3.68 & 5.10 & 6.23 \\
\hline Geodesic distance (in mm) & 0.73 & 1.19 & 2.14 & 1.03 & 1.41 & 1.51 \\
\hline
\end{tabular}

from the two validation experiments respectively. The quantitative validations demonstrate that our method provides an accurate and robust co-registration of the angiography and IVUS images.

\section{Conclusion}

To our knowledge, this is the first reported system to provide automatic and robust tracking and registration of angiography and IVUS images. The experiments demonstrate its effectiveness in fusing the two imaging modalities.

We hope that this work will establish a useful reference for further research in this exciting field. The developed approach can be adapted to 3D when using biplane angiography and also be used in combination with other intravascular imaging modalities such as optical coherence tomography (OCT).

\section{References}

1. Bruining, N., de Winter, S., Serruys, P.W.: Intravascular ultrasound registration/integration with coronary angiography. Cardiology Clinics 27(3), 531-540 (2009)

2. Rieber, J., Prummer, S., Schmidt, M., Rittger, H.: A novel method for direct correlation of angiography and endovascular diagnostic tools in human coronary arteries in vivo. J. Am. Coll. Cardiol. 55, A217.E2065 ( (2010)

3. Tu, S., Holm, N.R., Koning, G., Huang, Z., Reiber, J.H.C.: Fusion of 3D QCA and IVUS/OCT. Intl. J. Cardiovasc Imaging (2011)

4. Tu, Z.: Probabilistic boosting-tree: Learning discriminative models for classification, recognition, and clustering. In: ICCV, pp. 1589-1596 (2005)

5. Wahle, A., Prause, G., Birgelen, C.V., Erbel, R., Sonka, M.: Fusion of angiography and intravascular ultrasound in vivo: establishing the absolute 3-D frame orientation. IEEE Trans. on Biomedical Engineering (1999)

6. Wang, P., Chen, T., Zhu, Y., Zhou, S.K., Comaniciu, D.: Robust guidewire tracking in fluoroscopy. In: CVPR (2009)

7. Wang, P., Liao, W.-s., Chen, T., Zhou, S.K., Comaniciu, D.: Graph based interactive detection of curve structures in 2D fluoroscopy. In: Jiang, T., Navab, N., Pluim, J.P.W., Viergever, M.A. (eds.) MICCAI 2010. LNCS, vol. 6363, pp. 269277. Springer, Heidelberg (2010) 